\title{
Philosophiques
}

\section{Le non-conséquentialisme et l'universalisabilité}

\section{Philip Pettit}

Volume 27, numéro 2, automne 2000

URI : https://id.erudit.org/iderudit/004968ar

DOI : https://doi.org/10.7202/004968ar

Aller au sommaire du numéro

Éditeur(s)

Société de philosophie du Québec

ISSN

0316-2923 (imprimé)

1492-1391 (numérique)

Découvrir la revue

Citer cet article

Pettit, P. (2000). Le non-conséquentialisme et l'universalisabilité. Philosophiques, 27(2), 305-322. https://doi.org/10.7202/004968ar

\section{Résumé de l'article}

Si les non-conséquentialistes veulent adhérer à l'exigence d'universalisabilité, alors ils devront adopter une prise de position étonnamment relativiste. Non seulement vont-ils affirmer, dans une veine familière, que les prémisses invoquées dans l'argumentation morale n'ont de force que relative à l'agent, c'est-à-dire qu'elles peuvent impliquer l'usage d'un indexical — comme dans la considération que cette option-ci ou celle-là favoriserait mes engagements, me délesterait de mes devoirs ou bénéficierait à mes enfants - et qu'elles ne peuvent fournir de raisons qu'à l'agent indexicalement pertinent, moi-même en l'occurrence. Ils devront aussi interpréter la considération invoquée dans les conclusions morales typiques, selon laquelle telle ou telle option est juste ou devrait être choisie ou peu importe, comme n'ayant elle-même qu'une force relative à l'agent.
Ce document est protégé par la loi sur le droit d'auteur. L'utilisation des services d'Érudit (y compris la reproduction) est assujettie à sa politique d'utilisation que vous pouvez consulter en ligne.

https://apropos.erudit.org/fr/usagers/politique-dutilisation/ 


\title{
Le non-conséquentialisme et l'universalisabilité
}

\author{
PHILIP PETTIT \\ Australian National University \\ Pnp@coombs.anu.edu.au
}

RÉSUMÉ. - Si les non-conséquentialistes veulent adhérer à l'exigence d'universalisabilité, alors ils devront adopter une prise de position étonnamment relativiste. Non seulement vont-ils affirmer, dans une veine familière, que les prémisses invoquées dans l'argumentation morale n'ont de force que relative à l'agent, c'est-à-dire qu'elles peuvent impliquer l'usage d'un indexical — comme dans la considération que cette option-ci ou celle-là favoriserait mes engagements, me délesterait de mes devoirs ou bénéficierait à mes enfants - et qu'elles ne peuvent fournir de raisons qu'à l'agent indexicalement pertinent, moi-même en l'occurrence. Ils devront aussi interpréter la considération invoquée dans les conclusions morales typiques, selon laquelle telle ou telle option est juste ou devrait être choisie ou peu importe, comme n'ayant elle-même qu'une force relative à l'agent.

ABSTRACT. - If non-consequentialists are to embrace the requirement of universalisability, then they will have to strike a surprisingly relativistic stance. Not only will they say, in familiar vein, that the premises adduced in moral argument may only be agent-relative in force: that is, may involve the use of an indexical as in the consideration that this or that option would advance $m y$ commitments, discharge my duty, or benefit my children — and may provide reasons only for the indexically relevant agent: in this case, me. They will also have to construe the consideration adduced in typical moral conclusions, to the effect that this or that option is right or ought to be chosen or whatever, as itself only agent-relative in force.

Si les non-conséquentialistes veulent adhérer à l'exigence d'universalisabilité, alors ils devront adopter une prise de position étonnamment relativiste. Non seulement vont-ils affirmer, dans une veine familière, que les prémisses invoquées dans l'argumentation morale n'ont de force que relative à l'agent, c'est-à-dire qu'elles peuvent impliquer l'usage d'un indexical — comme dans la considération que cette option-ci ou celle-là favoriserait mes engagements, me délesterait de mes devoirs ou bénéficierait à mes enfants - et qu'elles ne peuvent fournir de raisons qu'à l'agent indexicalement pertinent, moi-même en l'occurrence. Ils devront aussi interpréter la considération invoquée dans les conclusions morales typiques, selon laquelle telle ou telle option est juste ou devrait être choisie ou peu importe, comme n'ayant elle-même qu'une force relative à l'agent. C'est en tout cas ce que je vais soutenir.

Développons un peu cette accusation : dire qu'une option est juste, comme dire qu'une option est prudente, référera à une propriété lorsque mes actions sont en cause, à une autre lorsque vos actions sont en cause, et le carac- 
tère juste (rightness) " de l'option fournira une raison pour préférer que l'option soit choisie seulement pour la personne désignée. J'aurai une raison de préférer que l'on choisisse l'option qui est juste relativement à moi, tout comme j'aurai une raison de préférer que l'on choisisse ce qui est prudent relativement à moi. Mais bien que vous puissiez être d'accord pour dire que les choix sont justes ou prudents selon cette interprétation - c'est-à-dire relativement à moi cela ne vous donne aucune raison de préférer vous-même que l'un ou l'autre de ces choix soit fait. La considération du caractère juste, tout comme la considération de la prudence, aura une force justificatrice (reason-giving force) seulement pour l'agent en fonction duquel elle est interprétée.

Cet essai tente d'établir que les non-conséquentialistes peuvent adhérer à l'universalisabilité seulement au prix de prendre le prédicat «juste » et ses semblables comme étant relatifs à l'agent en ce sens, ou seulement au prix d'adopter une tactique équivalente. Le prix, dans un vocabulaire différent ${ }^{1}$ mais potentiellement trompeur, est que les non-conséquentialistes devront assumer non seulement la relativité des raisons morales en fonction de l'agent, mais aussi la relativité des jugements moraux en fonction de l'évaluateur. Je n'affirme pas que ce prix est absolument prohibitif et je ne suggère pas que mon argumentation à ce sujet est entièrement originale. Cependant, je suis persuadé que le prix est substantiel, inexorable et insuffisamment évalué.

Mon essai se divise en cinq sections. Premièrement, je présente brièvement le conséquentialisme et le non-conséquentialisme. Ensuite, je caractérise et je motive l'exigence d'universalisabilité. Dans la troisième section, je décris un problème que soulève cette exigence pour le non-conséquentialisme, et dans la quatrième, j'ébauche la solution que, selon moi, les non-conséquentialistes devraient endosser, en mettant l'accent sur le relativisme qu'elle implique. Enfin, dans la dernière section, je démontre que deux autres solutions que les non-conséquentialistes pourraient envisager ont essentiellement le même effet de relativisation.

* Comme nous traduisons par « juste » le terme « right» et que le terme « justesse » ne traduit pas fidèlement la connotation morale de « rightness », nous traduisons « rightness " par « caractère juste ». N.D.T.

1. Je n'aime pas le vocabulaire de la relativité propre à l'évaluateur dans ce contexte-ci, pour la raison suivante. La relativisation de «juste » discutée dans cet essai relativise la condition de vérité pour les affirmations au sujet du caractère juste et leur force justificative, mais elle ne relativise pas leur valeur de vérité. Ainsi, tandis que les conditions de vérité de «Ceci est juste " dans mes propres mots peuvent être différentes de ses conditions de vérité dans les vôtres - et tandis qu'elles peuvent fournir une raison pour ma préférence à moi seulement il y a encore un fait non relatif, celui de savoir si la proposition dans mes mots est vraie, et cela est un fait sur lequel vous et moi pouvons nous entendre. S'exprimer en termes de relativité propre à l'évaluateur peut suggérer une relativité de la valeur de vérité - quelque chose dont la cohérence est douteuse - et non pas juste une relativité de la condition de vérité. 


\section{Le conséquentialisme et le non-conséquentialisme ${ }^{2}$}

Le non-conséquentialisme revêt plusieurs formes. Les kantiens affirment que les agents devraient agir en vertu de l'impératif catégorique ou devraient toujours traiter les autres comme des fins en soi. Les éthiciens de la vertu disent que leur comportement devrait refléter certaines vertus. Les déontologistes soutiennent qu'ils devraient s'acquitter de certains devoirs : ils devraient dire la vérité, tenir leurs promesses, être non violents, etc. Les théoriciens des droits soutiennent qu'ils devraient respecter certains droits qui protègent les autres contre eux. Les contractualistes affirment qu'ils devraient se conformer à des principes dont personne ne peut raisonnablement s'opposer au fait qu'ils sont les bases de la vie sociale. Les théoriciens de l'obligation spéciale disent qu'ils devraient agir d'une façon particulière à l'égard de ceux à qui ils sont attachés. Et ainsi de suite. ${ }^{3}$

Quel est le point commun entre ces positions ? Tous les non-conséquentialistes parlent, à quelque niveau d'abstraction que ce soit, de ce que n'importe quel agent doit faire ou être : en ce sens, ils sont universalistes. Tous les non-conséquentialistes prescrivent les mêmes structures neutres de comportement, de psychologie ou de relations avec les autres : les gens agissent selon l'impératif catégorique, manifestent certaines vertus, respectent les droits des personnes à leur charge, cultivent leurs amitiés, et ainsi de suite ; les structures sont neutres au sens où elles peuvent être comprises de la même façon par tous. Et tous les non-conséquentialistes affirment que la chose juste à faire pour un agent est d'instancier ces structures - dans la mesure où elles sont co-instanciables dans leur propre comportement, leur psychologie ou leurs relations avec les autres. Ils soutiennent en particulier que c'est la chose à faire même si instancier une structure dans leur propre vie signifie, à cause de la perversité des circonstances dans lesquelles se trouve l'agent, que la structure sera moins pleinement réalisée dans le monde pris dans sa totalité. J'aurai une raison relative à l'agent pour instancier la structure dans ma vie, et vous dans la vôtre, mais ni vous ni moi n'avons besoin d'avoir une raison neutre par rapport à l'agent de vouloir l'instanciation générale de la structure par les autres agents. ${ }^{4}$

2. La présentation du conséquentialisme et du non-conséquentialisme offerte ici - à strictement parler, le conséquentialisme et le non-conséquentialisme au sujet des actions s'inspire de travaux précédents, et plus récemment de ma contribution à Baron, Pettit et Slote, 1997. Le problème que je soulève pour le non-conséquentialisme est aussi soulevé dans cette contribution, quoique sous une forme quelque peu différente.

3. Je mets ici l'accent sur les prescriptions que les non-conséquentialistes soutiennent. Je n'affirme rien sur le fait que, parce qu'elles ne couvrent pas tous les aspects de l'action et de la vie, les prescriptions soutenues impliquent souvent des permissions correspondantes.

4. Il y a trois points à noter. Premièrement, la représentation que j'offre ici n'est pas la seule possible, mais cela n'affecte aucun aspect de mon argumentation, car les autres représentations que j'envisage sont distinctes seulement au niveau de la notation (voir ma contribution à Baron, Pettit et Slote, 1997). Deuxièmement, alors que je parle ici du fait que les gens instancient une structure dans leur comportement et dans leurs relations, j'ai parlé dans mon travail précédent de respecter la structure (Pettit, 1991). Et troisièmement, je considère ici 
Le conséquentialisme comme je l'entends fait une affirmation double. Premièrement, il affirme qu'il y a certaines valeurs potentiellement partagées au moyen desquelles les états de chose peuvent être classés, quoique peut-être pas complètement ; elles peuvent être des structures neutres par rapport au comportement humain, à la psychologie ou aux relations avec les autres, du même type que le non-conséquentialiste les prescrit, ou elles peuvent être des structures résultantes neutres (neutral outcome-patterns) qui sont plus éloignées de l'activité de l'agent, c'est-à-dire des structures telles que la maximisation du bonheur humain ou la protection de la nature sauvage. ${ }^{5} \mathrm{Et}$, deuxièmement, le conséquentialisme maintient que le choix juste à faire pour un agent dans toute décision est l'un de ces choix, en supposant qu'il y en ait au moins un - en supposant que le classement incomplet ne pose pas problème - qui promeut la réalisation globale de telles valeurs ou structures : nous n'avons pas besoin de trancher à propos de ce qu'est promouvoir des valeurs, bien qu'à première vue, cela pourrait correspondre à maximiser la valeur attendue.

Le conséquentialisme pris dans ce sens est une doctrine très ouverte. Il ne dit pas quelles sont les valeurs pertinentes. Il ne doit pas se résumer, par exemple, à l'utilitarisme; il peut même adopter comme valeurs plusieurs des structures recommandées par les non-conséquentialistes. Et le conséquentialisme pris dans ce sens laisse ouverte la question de savoir quelles habitudes quotidiennes de prise de décision devraient être cultivées par les agents (Pettit et Brennan, 1986 ; Railton, 1984). Il n'implique pas, par exemple, que l'agent devrait calculer chaque choix en se référant aux valeurs pertinentes. Un tel calcul pourrait être bien moins efficace pour la promotion de valeurs pertinentes qu'une politique voulant que l'on suive certaines routines dans la prise de décision, par exemple, une politique voulant que l'on s'ajuste sur l'impératif catégorique, ou sur ce que la manifestation des vertus requiert, ou sur les droits évidents que les autres peuvent revendiquer.

Mais aussi variées que puissent être les formes du non-conséquentialisme, et aussi ouverte que puisse être cette caractérisation du conséquentialisme, la différence entre les deux approches est évidente. Alors que les nonconséquentialistes soutiennent que certains structures doivent être instanciées dans le comportement et les relations de l'agent, même si cela signifie que leur réalisation globale n'est pas ainsi promue, le conséquentialisme inverse cet ordre. Il affirme que certaines structures doivent être promues,

seulement le genre de non-conséquentialisme qui requiert qu'un agent-à-travers-le-temps instancie certaines structures ; une version plus stricte requerrait qu'un agent-à-un-temps-donné instancie toujours ces structures, même si cela signifiait que la structure aurait été moins bien réalisée à travers la vie de l'agent prise globalement.

5. Alors que je suppose que toutes les structures du type que les non-conséquentialistes pensent que l'agent devrait instancier dans sa vie peuvent être prises comme des valeurs neutres à être promues, je ne suis pas enclin à penser que toutes les structures du type que les conséquentialistes pensent devoir être promues peuvent être prises comme des structures devant être instanciées dans la vie de l'agent. Voir McNaughton et Rawling, 1992. 
même si cela signifie que l'agent échouera à instancier des structures correspondantes dans sa propre vie.

La différence entre les deux approches est particulièrement évidente dans des cas simples où seulement une structure ou une valeur est considérée pertinente, et où il n'y a pas de désaccord au sujet de l'importance relative de différentes valeurs. Considérons ainsi des cas où seule la valeur de non-violence est supposée pertinente. Le pacifiste non-conséquentialiste affirme que la non-violence doit être instanciée par les agents. Son double conséquentialiste affirme que l'on doit promouvoir globalement la non-violence, même si cela signifie que l'on doit instancier la violence, par exemple en faisant la guerre afin d'éliminer toutes les guerres. Dans la pratique, les deux sortes de pacifistes feront souvent des recommandations convergentes, comme les Quakers non conséquentialistes convergèrent avec Bertrand Russell dans leur opposition à la Première Guerre mondiale. Mais il y a toujours une possibilité de divergence, comme bien sûr les Quakers et Russell divergèrent quant à leurs attitudes face à la Deuxième Guerre mondiale.

\section{L'exigence d'universalisabilité}

Chaque prescription concernant ce que devrait faire un agent devrait pouvoir être universalisée, afin qu'elle ne s'applique pas seulement à cet agent particulier, non plus qu'à cet espace, ce temps ou ce contexte particuliers, etc. ${ }^{6}$ C'est du moins ce que nous supposons en général dans notre raisonnement moral. Si nous pensons qu'il est juste pour un agent dans une certaine circonstance d'agir d'une certaine façon, mais injuste pour un autre, alors nous nous engageons à ce qu'il y ait des différences descriptives additionnelles entre les deux cas, en particulier une différence d'ordre non particulier ou universel.

Par conséquent, si nous affirmons qu'un agent A devrait choisir l'option $\mathrm{O}$ dans des circonstances $\mathrm{C}$ (qui peuvent porter sur le caractère de l'agent, le comportement des autres, le genre de conséquences présentes et ainsi de suite) alors nous supposons que quelque chose de similaire vaudrait pour tout agent placé dans des circonstances similaires. Nous ne pensons pas que l'identité particulière de l'agent $\mathrm{A}$ est pertinente pour déterminer ce que A devrait faire, pas plus que nous ne pensons que la localisation particulière ou la date sont pertinentes à cet égard. Bien sûr, en faisant une supposition à propos de ce qui vaut pour n'importe quel agent dans des circonstances de type $\mathrm{C}$, nous ne nous engageons peut-être à rien qui ait une portée très générale. Il se peut, en ce qui concerne ce que requiert l'exigence d'universalisabilité, que les circonstances de type $\mathrm{C}$ soient très spécifiques, tellement spécifiques en fait qu'il est probable qu'aucun autre agent n'y sera jamais confronté.

6. Rabinowicz, 1979. 
Il n'est pas difficile de voir comment le conséquentialisme est censé relever le défi de l'universalisabilité. Supposons que j'accepte la doctrine conséquentialiste et que je croie qu'un agent, dans ses circonstances particulières $\mathrm{C}$, devrait choisir une option O. Plus simplement, supposons que je sois moimême cet agent et qu'en tant qu'adepte du conséquentialisme, je pense à propos de moi-même que je devrais faire $\mathrm{O}$ dans $\mathrm{C}$. Si cette option est réellement juste selon mes raisons conséquentialistes, alors ce sera à cause des valeurs neutres qu'elle promeut. Mais si ces valeurs neutres font de O l'option juste pour moi dans ces circonstances, elles en feront par conséquent l'option juste pour n'importe quel agent dans de telles circonstances. Ainsi je peux facilement coupler la prescription à laquelle ma croyance dans le conséquentialisme me conduit avec ma croyance dans l'universalisabilité. Je peux aisément universaliser mon auto-prescription pour en faire une prescription qui vaut pour n'importe quel agent arbitraire dans des circonstances similaires.

Je dois faire en passant un commentaire au sujet de la forme de la prescription que le défi de l'universalisabilité me forcera à endosser. Je n'ai pas besoin de penser qu'il est juste que dans les circonstances pertinentes, tout agent fasse $\mathrm{O}$; cela suggère un engagement à une structure collective de comportement. Je serai seulement obligé de penser, en considérant chaque personne prise individuellement ou dans une optique distributive, qu'il est juste que chaque agent, dans ces circonstances, fasse $\mathrm{O}$. Admettons que faire $\mathrm{O}$ dans $\mathrm{C}$ consiste à nager afin de secourir un enfant en danger dans l'eau. L'universalisabilité ne m'obligerait pas à penser qu'il est juste que tout le monde nage à la rescousse d'un enfant dans une telle situation ; plusieurs personnes pourraient se trouver dans les environs et, s'ils se jetaient tous à l'eau, ils contrecarreraient mutuellement leurs efforts. La situation requiert seulement que je pense, comme nous le disons familièrement, qu'il est juste que quiconque nage à la rescousse de l'enfant, personne n'étant exempté de cette prescription individuelle et non collective (même s'ils font effectivement face à une exigence collective de décider qui en particulier ira nager). ${ }^{7}$

Voilà donc la manière directe par laquelle le conséquentialisme peut accommoder l'universalisabilité. Mais comment le défi d'universalisabilité peut-il être relevé par les théories non conséquentialistes ? Selon la théorie

7. La différence en est une de portée. Je n'ai pas à affirmer pour tout $\mathrm{X}$, que $\mathrm{X}$ devrait faire $\mathrm{O}$ dans des circonstances de type $\mathrm{C}$. Plutôt, pour tout $\mathrm{X}$, j'affirme que $\mathrm{X}$ devrait faire $\mathrm{O}$ dans des circonstances de type C. Cela aide à expliquer pourquoi l'universalisation de Hare est différente de la généralisation kantienne (voir Hare, 1981). Remarquez toutefois que si les circonstances de type C sont toujours assurées d'être spécifiées d'une façon qui est intuitivement complète - comme elles ne seront que rarement spécifiées dans la pratique - alors les deux prescriptions universelles peuvent être maintenues sans la suggestion qu'une structure collective est prescrite. Nous pourrions défendre volontiers l'universalisation qui donne une portée large à « il est juste ", par exemple bien que l'on puisse trahir l'usage ordinaire en procédant ainsi -, si la spécification stipulait non seulement qu'il y a un enfant en danger dans l'eau, mais qu'il y a seulement un sauveteur potentiel dans les environs. Je suis reconnaissant à Earl Conee pour ses commentaires sur ce point. 
non conséquentialiste, le choix juste pour tout agent consiste à instancier une certaine structure, $\mathrm{P}$, qui peut être une structure de conformité à l'impératif catégorique, de manifestation de vertu, de respect des droits, d'honorer ses obligations spéciales, etc. Supposons que j'accepte une telle théorie et qu'elle me conduise à affirmer au sujet d'un agent — supposons à nouveau qu'il s'agit de moi - que je devrais choisir $\mathrm{O}$ dans les circonstances $\mathrm{C}$, ou que $\mathrm{O}$ est le choix juste pour moi dans ces circonstances. Puis-je affirmer de manière directe, comme je pourrais le faire dans le cadre d'une doctrine conséquentialiste, que seulement du fait que $\mathrm{O}$ est le choix juste pour moi (c'est-à-dire, dans ce cas-ci, qu'il implique l'instanciation de la structure P), il sera par conséquent le choix juste pour n'importe quel agent dans des circonstances de type C ? Je soutiendrai qu'une réponse aussi directe soulève des difficultés, et que celles-ci entraînent un problème pour le non-conséquentialisme.

\section{Un problème pour l'universalisation non conséquentialiste}

Supposons que j'affirme effectivement, de façon directe, que la structure $\mathrm{P}$ requiert non seulement que je fasse $\mathrm{O}$ dans $\mathrm{C}$, mais aussi que, pour tout agent, cet agent devrait aussi faire $\mathrm{O}$ dans $\mathrm{C}$. Supposons que j'affirme, en effet, qu'il est juste pour moi de faire $\mathrm{O}$ dans $\mathrm{C}$ seulement s'il est juste pour n'importe quel agent X de faire $\mathrm{O}$ dans $\mathrm{C}$. Quel que soit ce qui rend juste le fait que je fasse $\mathrm{O}$ dans $\mathrm{C}$, cela rend également juste le fait que tout agent fasse $\mathrm{O}$ dans $\mathrm{C}$. Comme je vais le montrer maintenant, cette réponse, du fait que je suis un penseur non conséquentialiste, me causera des problèmes.

Juger qu'une action est juste implique l'approbation de l'acte et nous donne une raison normative de le préférer. Imaginez des gens disant penser que leur acte de faire une chose ou une autre, ou même que l'acte d'une autre personne faisant une chose ou une autre, était le choix juste, et qui alors nous communiquaient qu'ils l'approuvent. La question ne se poserait-elle pas de savoir s'ils savaient ce qu'ils disaient, s'ils avaient poursuivi en ajoutant qu'ils ne pensaient pas qu'ils avaient une bonne raison de préférer que cette action ait lieu plutôt qu'elle n'ait pas lieu ? Si le jugement sur le caractère juste doit jouer son rôle distinct dans l'examen ou la classification des actions (c'est-à-dire s'il doit faire le lien avec l'approbation d'une façon intuitive), alors on doit considérer qu'il fournit une raison normative de préférer que l'action ait lieu, et ce, peu importe s'il motive ou non la personne qui juge.

Lorsque je pense qu'il est juste que je fasse $\mathrm{O}$ dans $\mathrm{C}$, je m'engage par conséquent à ce qu'il y ait une raison normative pour que je préfère faire $\mathrm{O}$. Et lorsque j'affirme qu'il est juste que quiconque doive faire $\mathrm{O}$ dans des circonstances de type C, alors je m'engage (encore une fois à cause de la force justificatrice de la notion de caractère juste) à ce qu'il y ait une raison normative pour soutenir une préférence plus large. Je m'engage à ce qu'il y ait une raison normative à ce que je préfère que, pour n'importe quel agent, il fasse $\mathrm{O}$ dans les circonstances de type $\mathrm{C}$. 
Le problème avec ces raisons et ces engagements, toutefois, c'est qu'ils peuvent se séparer. En effet, il sera souvent possible que, de manière perverse, la meilleure façon pour moi de satisfaire la préférence que, pour tout agent arbitraire $\mathrm{X}$, cet agent fasse $\mathrm{O}$ dans des circonstances de type $\mathrm{C}$ soit de choisir moimême non-O dans ces circonstances. ${ }^{8}$ Choisir moi-même non-O signifie qu'il y a une personne, moi, en vertu de qui la préférence générale n'est pas satisfaite, mais dans les circonstances perverses, cela signifiera qu'il y a plus d'agents ou d'actions en vertu desquels elle sera satisfaite qu'il y en aurait eus si j'avais choisi O. Des circonstances perverses de ce type ne sont pas que des possibilités abstraites, car ce qu'un agent fait peut facilement affecter les motivations ou les opportunités des autres d'une façon qui génère de la perversité. La meilleure façon d'amener les gens à renoncer à la violence est peut-être de s'en charger soi-même et de menacer d'opposer une résistance à leur violence ; la meilleure façon de faire en sorte que les gens aident leurs enfants est peut-être de faire du prosélytisme et ne pas prêter l'attention voulue aux siens. Plus généralement, la meilleure façon de promouvoir l'instanciation de la structure P, lorsque celle-ci est la structure fondamentale à laquelle on prête une allégeance non conséquentialiste, est peut-être de faire fi soi-même de cette structure.

Comment puis-je éviter la conclusion que dans une telle situation perverse, je devrais promouvoir l'instanciation globale de ma structure préférée, même au prix de ne pas l'instancier moi-même ? Autrement dit, comment puis-je conserver ma foi dans l'engagement non conséquentialiste à l'égard du caractère juste de l'instanciation de $P$, même où cela signifie que la réalisation globale de la structure ne parvient pas à être ce qu'elle aurait pu être ?

Il semble difficilement plausible d'affirmer que les raisons normatives portant sur les préférences au sujet de mes propres choix ont préséance sur les raisons normatives portant sur les préférences au sujet de la façon dont les gens se comportent de façon générale. Les deux types de raison sont présentes dans le langage commun sur ce qu'est le choix juste ou sur ce qui devrait être fait. ${ }^{9}$ Et il irait sûrement contre l'esprit d'universalisabilité — l'esprit dans lequel je nie que mon identité propre et particulière est importante pour la prescription

8. Pourquoi est-ce que j'affirme que cela tient souvent, et non pas toujours ? Parce que, tel que mentionné en effet dans la note précédente, certaines options peuvent être caractérisées d'une façon qui ne laisse pas de place à la possibilité perverse. Je pense ici à une option telle que nager à la rescousse d'un enfant en train de se noyer lorsque personne d'autre ne le fait.

9. Un non-conséquentialiste pourrait songer à argumenter ici que la notion de justice a une force justificatrice pour quelqu'un qui fait un jugement sur le caractère juste seulement quand le jugement porte sur sa propre action. Mais il est difficile de donner un sens à cette tactique sans l'indexicalisation — ou d'autres approches équivalentes — introduite plus loin. Si l'auto-prescription me donne raison de préférer ce que je me prescris, alors la prescription pour des agents en général semblerait me donner raison de préférer ce que je prescris pour de tels agents. Les non-conséquentialistes attirés par la tactique envisagée ici ont besoin d'une théorie de la responsabilité au sein de laquelle une personne se voit attribuer une raison pour l'action par une raison de préférer que X fasse quelque chose seulement lorsqu'elle est X. Mais il est difficile de voir comment concevoir une théorie plausible de la responsabilité (elle-même de 
que je défends - d'affirmer qu'une préférence raisonnée au sujet de ce que moi-même je devrais faire ne devrait pas être sensible à une préférence similairement raisonnée à l'égard de ce que les gens en général font (ce que l'agent arbitraire, X, fait) dans le type de circonstances dont il est question. Cette considération devient particulièrement éloquente lorsque nous nous souvenons que la satisfaction d'une préférence individuelle peut signifier que seulement une personne pose l'action approuvée, alors que sa non-satisfaction peut signifier qu'un grand nombre pose cette action. Comment justifier la satisfaction d'une préférence individuelle sans privilégier le moi et rejeter l'esprit d'universalisabilité ?

Bien sûr, agir afin de satisfaire une préférence portant sur la façon dont les autres se comportent va souvent de pair avec le fait d'être un fouineur et de bafouer des valeurs indépendantes qui portent sur des relations interpersonnelles, comme les valeurs associées au fait d'être un concitoyen respectueux, un collègue ou un ami. Dans ces cas, la pertinence de ces valeurs constituerait certainement un argument (qui peut être formulé en termes conséquentialistes ou non-conséquentialistes) contre le fait d'agir, ou du moins d'agir de certaines façons, en fonction de la préférence portant sur la façon dont les autres agissent. Mais le présent raisonnement fait abstraction de telles considérations. Il soulève la question de savoir de quelle manière l'agent non-conséquentialiste devrait réagir à la reconnaissance de trois aspects : premièrement, qu'il y a une bonne raison de préférer à la fois qu'il ou elle fasse $\mathrm{O}$ dans $\mathrm{C}$ et que l'agent arbitraire $\mathrm{X}$ fasse $\mathrm{O}$ dans $\mathrm{C}$; deuxièmement, que ces préférences ne peuvent pas être satisfaites simultanément; et troisièmement, pouvons-nous maintenant ajouter, qu'il n'y a pas d'autres valeurs pertinentes qui plaideraient contre le fait de satisfaire soit la préférence dirigée vers soi-même, soit la préférence plus générale. Cette question demeure encore, et l'argument suggère encore que le non-conséquentialiste sera peut-être obligé, par l'esprit d'universalisabilité, de lui donner une réponse typiquement conséquentialiste.

Le résultat est que si, en tant que théoricien non-conséquentialiste, j'universalise de manière directe la prescription voulant que, dans une certaine situation, je devrais instancier une structure favorisée $\mathrm{P}$, alors la prescription à laquelle je m'engage dès lors (et qui dit que dans cette situation, tout $\mathrm{X}$ devrait instancier la structure P) peut m'obliger à revoir mon auto-prescription de départ. J'ai des raisons équivalentes de préférer à la fois que j'instancie $P$ et que tout agent instancie P (cette raison est exprimée par l'usage du mot « juste » ou « devrait » dans chaque cas), et l'esprit d'universalisabilité empêche que je me traite d'une façon spéciale. Ainsi, si les préférences sont incohérentes dans une certaine situation - si le choix se trouve entre mon instanciation solitaire de

caractère non conséquentialiste) qui ferait l'affaire. Et de toute façon, une telle théorie de la responsabilité aurait un effet de relativisation similaire à celui qui guette la stratégie d'indexicalisation-décentrement considérée plus loin. 


\section{Philosophiques / Automne 2000}

$\mathrm{P}$, par exemple, et le fait que j'agisse plutôt de façon à ce que plusieurs autres instancient $\mathrm{P}$ - alors j'aurai une raison pour ne pas instancier P moi-même.

En tant que penseur aspirant au non-conséquentialisme, mon affirmation initiale doit avoir été que le but est d'instancier P dans ma propre vie, pas de le promouvoir généralement. Mais j’admets les affirmations générales de la structure P lorsque j'universalise de façon directe : je prescris une conformité générale à cette structure, non pas une conformité dans mon propre cas. Ainsi, il semble maintenant que ce que je dois penser est que cette conformité générale doit être promue, même si cela signifie que je n'instancie pas moi-même la structure dans mon propre comportement, ma propre psychologie ou mes propres relations. Il semble que ce à quoi je dois adhérer, en fait, soit un conséquentialisme dans lequel la conformité à la structure P est la valeur ultime devant être promue.

Le genre de conséquentialisme envisagé ici n'est pas une doctrine barbare que l'on doit imposer à certains non-conséquentialistes avoués. C'est un conséquentialisme dans lequel le but est la conformité maximale à une structure réflexive, c'est-à-dire à une structure par laquelle, pour tout X, X instancie une certaine structure dans son comportement, sa psychologie ou ses relations. X ne traite pas les autres personnes seulement comme des moyens, $\mathrm{X}$ démontre un certain modèle de vertu, $\mathrm{X}$ tient ses promesses, $\mathrm{X}$ respecte les droits de ceux avec qui il entre en relation, et ainsi de suite. Il se peut bien que plusieurs non-conséquentialistes avoués soient en fait des conséquentialistes réflexifs en ce sens précis. Ils sont peut-être des conséquentialistes réflexifs qui pensent qu'il est extrêmement improbable que le fait de promouvoir la structure réflexive dont ils font l'éloge requerra un jour qu'on la bafoue et, par conséquent, ils n'ont que de très faibles motivations pour accentuer la dimension conséquentialiste de leur engagement.

Un exemple d'un conséquentialiste réflexif est la personne décrite par Robert Nozick comme étant un utilitariste de la règle : quelqu'un qui pense qu'un agent comme l'État devrait agir de façon à ce qu'il y ait plus plutôt que moins de satisfaction des droits, même si cela signifie qu'il bafouera lui-même certains droits ${ }^{10}$. Et un autre exemple est le théoricien du juste mérite qui pense que les cours devraient émettre occasionnellement des verdicts exemplaires, afin d'assurer que le régime du juste mérite soit promu, c'est-à-dire afin de minimiser le nombre de cas où les contrevenants s'en sortent impunément ${ }^{11}$. De tels penseurs se décrivent souvent comme des non-conséquentialistes, mais il est clair que ce à quoi ils adhèrent vraiment est un conséquentialisme dans lequel le but général est de promouvoir un certain régime de droit ou de mérite. ${ }^{12}$

10. Nozick, 1974, p. 28.

11. Braithwaite et Pettit, 1990.

12. Un autre exemple de conséquentialisme réflexif serait le conséquentialisme que David Cummiskey (1996) dérive à partir des principes kantiens, c'est-à-dire un conséquentialisme sous lequel ce qu'il faut maximiser est le traitement d'êtres rationnels comme des fins en soi et non seulement comme des moyens. 


\section{Une façon de sortir du problème}

Mais plusieurs non-conséquentialistes vont reculer devant l'idée d'adhérer au conséquentialisme réflexif. Dès lors, y a-t-il d'autres recours disponibles ? Y a-t-il une façon par laquelle un non-conséquentialiste peut endosser de façon cohérente la contrainte d'universalisabilité ?

La raison pour laquelle la réponse directe au défi de l'universalisabilité soulève un problème pour le non-conséquentialisme est celle-ci. Elle m'obligerait à penser que, tout comme il est juste que j'instancie P dans une certaine situation, alors, dans le même sens de "juste », il est juste que chacun instancie $\mathrm{P}$ dans ce type de situation. Elle m'obligerait à employer le même étalon d'évaluation - le même sens de «juste » ou « devrait » ou ainsi de suite - lorsque j'exprime la valeur que j'accorde à mon instanciation de $\mathrm{P}$ et à l'instanciation de $\mathrm{P}$ par qui que ce soit. La seule façon d'échapper à ce problème sera alors, pour le non-conséquentialiste, d'argumenter que c'est une méprise et qu'en universalisant le jugement particulier au sujet de ce qu'il est juste pour moi de faire - en le déplaçant vers un jugement au sujet de ce qui est juste pour chacun de faire - j'introduis un vocabulaire évaluatif qui ne m’engage pas de la même façon.

Or, il existe une stratégie simple par laquelle les non-conséquentialistes peuvent mettre en place cette solution. Dans le mode direct de l'universalisation, " Il est juste pour moi de faire $\mathrm{O}$ dans $\mathrm{C}$ » se transforme en « Il est juste pour tout $\mathrm{X}$ arbitraire de faire $\mathrm{O}$ dans $\mathrm{C}$ », où « juste » dans chaque cas, est utilisé pour exprimer le même type de prescription. Mais les non-conséquentialistes peuvent échapper au problème soulevé en argumentant que « juste » — ou " devrait », et ainsi de suite — n'exprime pas le même type de prescription dans les deux jugements.

Les non-conséquentialistes peuvent arriver à ce résultat en deux étapes. Premièrement, ils peuvent argumenter que lorsqu'une personne, A, affirme que quelque chose est juste, ou tout au moins qu'elle est juste pour elle, alors « juste » est indexical et est mieux représenté comme "justeA »; ce prédicat indexicalisé sert à identifier la propriété de satisfaire aux exigences de $\mathrm{A}$, tout comme le prédicat correspondant « justeB »- « juste » tel qu'utilisé par $\mathrm{B}$ - servirait à identifier la propriété de satisfaire aux exigences de $\mathrm{B}^{13} \cdot{ }^{14} \mathrm{Et}$ ensuite, en deuxième lieu, ils peuvent affirmer que la façon d'universaliser le jugement de A, «Il est justeA que je, A, fasse O dans $\mathrm{C}$ », n'est pas

i) $(\mathrm{X})(\mathrm{Il}$ est justeA que $\mathrm{X}$ fasse $\mathrm{O}$ dans $\mathrm{C}$ ) ; mais plutôt

ii) $(\mathrm{X})(\mathrm{Il}$ est justeX que $\mathrm{X}$ fasse $\mathrm{O}$ dans $\mathrm{C})$.

13. Dreier, 1990.

14. Sur le recours à l'indexicalisation et d'autres raisons pour l'invoquer, voir Humberstone, 1991. Voir en particulier p. 148-149, où il envisage une formulation de l'égoïsme éthique qui est parallèle à la formulation que j'envisage ici pour le non-conséquentialisme. 


\section{$316 \cdot$ Philosophiques / Automne 2000}

Alors que l'indexicalisation du prédicat «juste » laisse ouvertes ces deux options, endosser la deuxième signifie considérer le prédicat comme n'étant pas seulement indexical mais aussi, pourrait-on dire, décentré.

Si les non-conséquentialistes empruntent cette voie, alors ils peuvent échapper au mouvement qui les mène vers le conséquentialisme réflexif. Car en prenant « juste " - et "devrait » et "doit » et d'autres mots de ce genre - comme étant indexical, ils peuvent assurer que mon engagement à l'égard de ce que je fais moi-même a une prise sur moi, prise que mon engagement au sujet de ce que quiconque fait dans des circonstances similaires n'a pas nécessairement. Universaliser ne doit plus maintenant signifier admettre une prescription universelle implicite dans ma prescription au sujet de ce que je devrais faire moi-même. Cela doit seulement signifier reconnaître que, tout comme je prescris pour moi-même dans ma propre position, les autres ont aussi le droit de prescrire pour eux-mêmes dans leurs propres positions.

Dans cette approche, la question qui se pose lorsqu'une personne demande s'il est juste pour elle de faire un certain choix, comme la question soulevée lorsqu'elle demande s'il est prudent, varie d'une personne à l'autre. A demande si c'est justeA, B demande si c'est justeB et ainsi de suite. L'affirmation non-conséquentialiste est qu'il est justeA pour A d'instancier P dans la vie de A et, semblablement, qu'il est justeB pour B d'instancier P dans la vie de B. Ainsi, l'universalisation n'engage pas un non-conséquentialiste, A, à penser que n'importe quelle structure générale est justeA ; cet engagement ferait surgir le même problème qu'auparavant. Elle engage seulement $A$ à penser que, comme l'instanciation de $\mathrm{P}$ par A est justeA, l'instanciation de $\mathrm{P}$ par B est justeB, et ainsi de suite. ${ }^{15}$

Il est peut-être peu probable que l'on ait à passer par les deux étapes décrites - indexicaliser et décentrer « juste » - mais ce n'est sûrement pas une stratégie absurde à adopter pour les non-conséquentialistes. Lorsque nous utilisons le mot " prudent ", nous changeons effectivement souvent la référence indexicale du terme (ici, je veux dire prudent pour moi, là, je veux dire prudent pour vous), et il n'est donc pas incohérent d'affirmer que, lorsque je parle du caractère juste, ici relativement à mes actions, là relativement aux vôtres, l'index du prédicat change. ${ }^{16}$

15. Un tel non-conséquentialiste peut soutenir que pour tout $\mathrm{X}$ et $\mathrm{Y}$, s'il est justeX que $\mathrm{X}$ fasse quelque chose, alors il est justeY que $\mathrm{Y}$ permette à $\mathrm{X}$ de le faire ; l'important est qu'ils ne soutiennent pas que dans un tel cas il doit être justeY que $\mathrm{X}$ le fasse.

16. Mais remarquez que le genre de tactique envisagé ici n'est pas cohérent avec un point de vue expressiviste, comme étant distinct d'un point de vue cognitiviste. Car, selon l'expressivisme, «juste » dans la bouche du locuteur exprimera toujours les attitudes du locuteur et jamais les attitudes d'un agent arbitraire, $\mathrm{X}$; il servira toujours de manière parallèle à la façon dont « justeA » sert, selon ce que nous disons ici, jamais de manière parallèle à la façon dont « justeX » sert. James Dreier (1996) offrirait au non-cognitiviste une autre façon de sortir de cette difficulté, en utilisant l'approche décrite dans la prochaine section. Je n'ai pas beaucoup d'estime pour cette façon de s'en sortir, mais ce n'est pas l'endroit pour en débattre. 
Cependant, si les non-conséquentialistes sont obligés d'adopter cette stratégie, cela est très révélateur. Cela démontre que le non-conséquentialisme implique une relativisation contre-intuitive du caractère juste. Alors que les conséquentialistes soutiennent que la justification réussie d'un jugement moral doit toujours ramener les gens à des valeurs communes - elle doit démontrer que ce jugement est exigé par ces valeurs partagées -, les non-conséquentialistes abaissent les exigences que doit satisfaire la justification. Selon eux, tout ce que la justification doit faire, c'est démontrer à chaque personne à laquelle on s'adresse que le jugement posé et que l'action entreprise étaient raisonnables selon le point de vue de l'agent, et, en particulier, que c'était un équivalent du jugement et de l'action qu'elle aurait pu elle-même endosser dans son propre cas.

Selon cette approche, parler de ce qui est juste dans son propre cas n'équivaut jamais à parler d'une propriété commune. Cela équivaut seulement à employer un schème commun par lequel chaque participant peut référer à une propriété qui lui importe, c'est-à-dire la propriété d'être juste, telle qu'elle est indexée à leur position. Par conséquent, ma tentative d'argumenter que quelque chose que j'ai fait était juste n'est pas un exercice qui fait référence à des raisons communes. C'est une tentative de démontrer que les raisons en vertu desquelles l'action semblait acceptable pour moi, bien qu'elles ne soient pas nécessairement partagées par d'autres, sont tout au moins des raisons que les autres devraient comprendre. C'est une tentative d'exposer, non pas une identité dans les engagements évaluatifs, mais plutôt un isomorphisme de points de vue évaluatifs.

Considérons une analogie. Les patriotes se soucient tous de leur propre patrie et, dans cette mesure, ils ont quelque chose en commun. Mais la propriété qui émeut le patriote de chaque pays est différente des propriétés qui émeuvent les patriotes d'ailleurs : c'est la propriété de servir son pays particulier que chacun d'eux trouve émouvant, et non la propriété de servir le pays dans lequel on se trouve à être. De façon similaire, dans la formulation présente du non-conséquentialisme, la propriété invoquée pour justifier n'importe quel agent particulier sera une propriété différente de celle qui est invoquée dans la justification d'autres agents. Ce que de tels agents doivent avoir en commun alors ne doit pas être quelque chose de plus substantiel que la structure commune d'attachement qui caractérise les patriotes.

\section{D'autres façons de s'en sortir}

J'ai décrit un moyen par lequel les non-conséquentialistes peuvent échapper au problème qui leur est posé par l'exigence d'universalisabilité - l'échappatoire le plus simple, il me semble - et j'ai argumenté que cela engage le non-conséquentialisme à une relativisation contre-intuitive du caractère juste. Mais d'autres versions du non-conséquentialisme pourraient aussi affirmer qu'elles offrent un échappatoire à ce problème, et ma dernière 


\section{Philosophiques / Automne 2000}

question consiste à demander si elles impliquent le même genre de relativisation. Je crois que, d'une façon ou d'une autre, elles ont toutes cet effet de relativisation - sous cet angle, elles ne diffèrent pas de l'échappatoire d'indexicalisation-décentrement — et je voudrais défendre cette affirmation en considérant deux suggestions récentes parmi les plus élaborées.

James Dreier ${ }^{17}$ offre un premier exemple de la façon dont le non-conséquentialisme pourrait échapper au problème posé sans indexicaliser ou décentrer le prédicat juste. Selon cette approche (qu'il développe pour d'autres fins), nous devrions reconnaître trois affirmations :

1) affirmer qu'une action, comme tenir une promesse ou promouvoir le bonheur, est juste implique le fait de reconnaître une raison normative pour vouloir la propriété de tenir une promesse ou de promouvoir le bonheur, qui serait la propriété-cible de l'action ;

2) vouloir une telle propriété signifie vouloir l'instancier soi-même, c'est-à-dire vouloir être un teneur de promesse ou un promoteur du bonheur ;

3 ) et donc juger une action comme étant juste et approuver sa propriété-cible est cohérent avec le fait de ne pas reconnaître une raison normative de vouloir que les autres instancient cette propriété et qu'ils fassent l'action.

On ne voudra certainement pas que les autres instancient la propriété, suggère Dreier, dans ces cas pervers où le fait qu'ils l'instancient est incohérent avec le fait de l'instancier soi-même. Que les autres promeuvent le bonheur peut toujours être cohérent avec le fait de le promouvoir soi-même, mais que les autres tiennent leurs promesses ne doit pas nécessairement être cohérent avec le fait de tenir sa propre promesse. Et, toutes choses étant égales par ailleurs, on ne voudra pas que les autres tiennent leurs promesses on voudra qu'ils ne les tiennent pas - si cela signifie qu'on ne peut pas tenir les siennes. On décrira comme juste leur acte de tenir leurs promesses - on approuvera la propriété-cible - mais on ne voudra quand même pas que les autres tiennent leurs promesses. Et cela sera convenable, car le jugement que le respect de leurs promesses est juste, selon cette approche, ne fournit pas une raison normative pour vouloir qu'ils tiennent leurs promesses.

Selon cette approche, le jugement qu'une action est juste aura seulement de l'importance dans l'attribution de raison pour ce que l'on devrait désirer et faire soi-même. Cela se ramènera à un jugement selon lequel la propriété finale en question en est une qu'il est juste d'instancier et raisonnable de préférer, où cela signifie qu'il est raisonnable de préférer l'instancier soimême. Et ainsi, le jugement qu'une action est juste offrira uniquement une base pour préférer qu'on la fasse soi-même, là où c'est pertinent ; il n'aura pas d'influence sur la manière dont on devrait formuler des préférences au sujet des actions des autres.

17. Dreier, 1996, p. 419. 
Mais cette approche n'offre aucune consolation aux non-conséquentialistes, car elle porte en elle le même type de relativisation que la stratégie d'indexicalisation-décentrement décrite dans la dernière section. La stratégie d'indexicalisation-décentrement laisse en place la règle d'inférence par laquelle le caractère juste de deux actions ou plus - leur caractère juste dans le même sens - indique également qu'il y a une bonne raison de préférer que chacune soit effectuée, et ce sans tenir compte de l'identité de l'agent. Ainsi, en toute cohérence avec le fait de laisser cette règle d'inférence en place, elle indique que le non-conséquentialiste doit trouver un sens différent de ce qu'est le caractère juste dans des affirmations au sujet de ce qu'il est juste qu'un agent fasse lui-même et dans des affirmations au sujet de qu'il est juste qu'un agent arbitraire fasse. L'indexicalisation et le décentrement garantissent qu'un tel sens différent est disponible.

L'approche de Dreier consiste plutôt à réviser la règle d'inférence mentionnée. Elle construit les choses de sorte que l'observation qu'une action est juste - qu'elle réalise une propriété-cible approuvée par l'observateur n'offre jamais qu'une raison pour laquelle cette personne devrait préférer faire l'action ; elle ne lui donne jamais de raison spécifique de préférer que les autres la fassent. Cette construction implique la relativisation de la règle d'inférence pratique au moyen de laquelle les jugements au sujet du caractère juste fournissent des raisons pour la préférence, et ce d'une façon qui suit fidèlement la stratégie que nous avons décrite. Alors que cette stratégie relativiserait le jugement à propos du caractère juste lui-même et garderait une règle d'inférence ordinaire, cette construction atteint le même but en gardant le jugement ordinaire sur le caractère juste et en relativisant la règle d'inférence. Les deux approches invitent les non-conséquentialistes à relativiser l'importance du caractère juste; elles diffèrent seulement au sujet de l'endroit où l'on introduit la relativisation recommandée.

Une deuxième version du non-conséquentialisme qui pourrait sembler offrir un échappatoire au problème de l'universalisabilité est présentée dans une série d'essais par David McNaughton et Piers Rawling ${ }^{18}$. Ils suggèrent que les non-conséquentialistes attribuent une forme canonique aux jugements sur le caractère juste de sorte que, sous cette forme canonique, l'universalisation ne fait pas problème; elle ne génère pas un glissement vers le conséquentialisme.

La forme canonique qu'ils conseillent pour des jugements relativement simples portant sur ce qu'un agent est obligé de faire est la suivante : je devrais m'assurer (plus brièvement, "JD») que je fais $\mathrm{O}$ dans $\mathrm{C}$, ou schématiquement : (JD [je fais $\mathrm{O}$ dans $\mathrm{C}$ ]). Ils affirment que les non-conséquentialistes universaliseront ce jugement dans l'esprit d'une formulation correspondante : pour tout $\mathrm{x}$ arbitraire, $\mathrm{x}$ devrait s'assurer que $\mathrm{x}$ fait $\mathrm{O}$ dans $\mathrm{C}$; ou, encore schématiquement : $(\mathrm{x}) \mathrm{xD}[\mathrm{x}$ fait $\mathrm{O}$ dans $\mathrm{C}])$. Et, ce qui est 
d'importance cruciale, ils comprennent cette prescription universelle à son tour de telle sorte que je, le sujet universalisant — je, l'agent à qui est attribuée une raison normative par le jugement original de vouloir que je m'assure que je fais $\mathrm{O}$ dans $\mathrm{C}$ - ne se voit pas attribuer la même raison, dans l'acte d'universalisation, de vouloir qu'un $\mathrm{x}$ arbitraire s'assure qu'il fait $\mathrm{O}$ dans $\mathrm{C}$.

En imposant leurs formulations canoniques et leurs interprétations, McNaughton et Rawling régentent le langage de façon à ce que le problème que j'ai posé pour les non-conséquentialistes ne puisse pas être soulevé. Ce problème survient seulement si on nous accorde l'accès à un opérateur ou à un prédicat déontiques — « juste » ou « devrait » ou « doit » et ainsi de suite — qui peut servir à articuler ce que je veux en fonction d'une raison normative, même lorsque la chose en question concerne les actions des autres plutôt que les miennes. McNaughton et Rawling me permettent d'affirmer que, pour un $\mathrm{x}$ arbitraire, $\mathrm{x}$ devrait s'assurer que $\mathrm{x}$ fait $\mathrm{O}$ dans $\mathrm{C}$. Mais ils insistent pour que cela soit interprété de telle manière que le «devrait s'assurer » n'ait pas la même force que le « devrais m'assurer » dans « je devrais m'assurer que je fais $\mathrm{O}$ dans $\mathrm{C}$ ». Il n'exprime pas une raison normative de préférer qu'un $\mathrm{x}$ arbitraire s'assure que $\mathrm{x}$ fait $\mathrm{O}$ dans $\mathrm{C}$, de la façon dont il peut exprimer une raison normative de préférer que je m'assure que je fais $\mathrm{O}$ dans $\mathrm{C}$.

Toutefois, cette façon de régimenter le langage déontique équivaut effectivement au fait de prescrire la tactique d'indexicalisation-décentrement. Si nous étions obligés d'indexicaliser "juste » et de décentrer l'index dans les prescriptions universelles, alors nous serions obligés de nous conformer précisément au moule dans lequel McNaughton et Rawling voudraient nous forcer. A pourrait affirmer dans son propre cas qu'il est justeA qu'il fasse $\mathrm{O}$ dans $\mathrm{C}$, ou qu'il s'assure qu'il fait $\mathrm{O}$ dans $\mathrm{C}$. Et la seule universalisation disponible pour $\mathrm{A}$ serait d'affirmer que pour tout $\mathrm{x}$ arbitraire, il est justeX que $\mathrm{x}$ fasse $\mathrm{O}$ dans $\mathrm{C}$, ou que x s'assure qu'il fait $\mathrm{O}$ dans $\mathrm{C}$. Ainsi pourrait-on soutenir que, tandis que l'auto-prescription donne à $\mathrm{A}$ une raison normative de préférer qu'il fasse $\mathrm{O}$ dans $\mathrm{C}$, la prescription universelle ne donne pas de raison normative à $\mathrm{A}$ pour préférer que tout $\mathrm{x}$ arbitraire fasse $\mathrm{O}$ dans $\mathrm{C}$.

L'interprétation du non-conséquentialisme de Dreier imposerait le même genre de relativisation que la stratégie d'indexicalisation-décentrement, en localisant la relativisation dans un endroit différent, c'est-à-dire dans la règle d'inférence provenant du jugement sur le caractère juste plutôt que dans le contenu du jugement lui-même. L'interprétation offerte par McNaughton et Rawling aurait exactement le même effet de relativisation que ces stratégies, mais elle déguiserait cet effet comme le produit de ce qui, supposément, est la seule et unique façon correcte de formuler et d'interpréter les auto-prescriptions et les prescriptions universelles. Je pense que les non-conséquentialistes devraient préférer la stratégie d'indexicalisation-décentrement, parce qu'elle rend plus clair ce qui se passe et comment exactement ils arrivent à éviter le glissement de l'universalisabilité vers le conséquentialisme. Mais cela n'est pas une thèse que je souhaite établir maintenant. 
La leçon sur laquelle je veux insister, plutôt, est que, peu importe comment les non-conséquentialistes formulent le sens de leur position, ils doivent tôt ou tard adhérer à une sorte de relativisation caractéristique. Ils doivent penser que la justification morale ne peut pas être obligée de recourir, même au niveau le plus fondamental, aux valeurs que tous peuvent admettre en commun. Ils doivent penser que la meilleure et la seule sorte de justification qu'une personne puisse offrir aux autres pour les actions qu'elle a entreprises consiste à dire que c'est une sorte d'action que ces autres auraient trouvée justifiable s'ils avaient été dans la position de l'agent, et non pas que c'est la sorte d'action qui promeut quelque chose dont ils devraient se soucier en commun. Ils doivent penser que le mieux que les agents moraux puissent accomplir vers la compréhension mutuelle est le type de compréhension partagée que les patriotes de pays différents atteignent, lorsqu'ils reconnaissent que, bien que leurs soucis soient différents, et même peut-être en concurrence, ils sont du moins isomorphiques. Ils doivent penser, et peut-être ce parallèle est-il encore plus ciblé, que c'est le type de compréhension mutuelle que les égoïstes atteignent lorsqu'ils reconnaissent que, bien que leurs cœurs soient orientés vers des fins fort différentes, ils n'en palpitent pas moins au même rythme égocentrique ${ }^{19,20 .}$

\section{Bibliographie}

Baron, Marcia, Pettit, Philip, Slote, Michael, Three Methods of Ethics : A Debate, Oxford, Blackwell, 1997.

Braithwaite, John, Pettit, Philip, Not Just Deserts : A Republican Theory of Criminal Justice, Oxford, Oxford University Press, 1990.

Dreier, James, "Internalism and Speaker relativism ", Ethics, 101, 1990, p. 6-26.

Dreier, James, «Accepting Agent Centred Norms : A Problem for Noncognitivists and a Suggestion for Solving It », Australasian Journal of Philosophy, 1996, p. 409-421.

Hare, R.M., Moral Thinking : Its Levels, Method and Point, Oxford, Oxford University Press, 1981.

Humberstone, I.L., "Two Kinds of Agent-Relativity », Philosophical Quaterly, 41, 1991, p. 144-166.

19. J'aimerais remercier chaleureusement Don Reagan qui m'a défié à chaque étape de l'argumentation. Je remercie aussi les nombreux auditoires auxquels j'ai présenté cet essai : au Colloque de Cerisy, à La Trobe University, à la Monash University, à la University of Canterbury, Chrischurch, à l'Université de Montréal, à la Brown University et à la St Louis University. Les commentaires de Diane Proudfoot et Jack Copeland m'ont particulièrement aidé, ainsi que les discussions avec Lloyd Humberstone, Peter Railton, Piers Rawling, Michael Ridge et Michael Smith.

20. Cet article a été traduit de l'anglais par Éric Racine et Christine Tappolet. La version originale a été publiée dans The Philosophical Quaterly, 50, 2000, 175-190 
McNaughton, David, Rawling, Piers, "Agent-Relativity and the DoingHappening Distinction ", Philosophical Studies, 63, 1991, p. 167-85.

McNaughton, David, Rawling, Piers, "Honoring and Promoting Values ", Ethics, 102, 1992, p. 835-43

McNaughton, David, Rawling, Piers, "Value and Agent-Relative Reasons ", Utilitas, 7, 1995, p. 31-47.

Nozick, Robert, Anarchy, State, and Utopia, Oxford, Blackwell, 1974.

Pettit, Philip, "Consequentialism ", in A Companion to Ethics, Singer, P., dir., Oxford, Blackwell, 1991.

Pettit, Philip and Brennan, Geoffrey, "Restrictive Consequentialism ", Australasian Journal of Philosophy, 64, 1986, p. 438-55.

Rabinowicz, Wolodzimierz, Universalizability: A Study in Morals and Metaphysics, Dordrecht, Holland, Reidel, 1979.

Railton, Peter, "Alienation, Consequentialism, and the Demands of Morality ", Philosophy and Public Affairs, 13, 1984, p. 134-71. 